 \\ TATRA \\ MOUNTaiNS \\ Mathematical Publications
}

\section{A NOTE ON MEASURE EXTENSION PROBLEM OF $\ell$-GROUP-VALUED MEASURES}

\author{
SuRJit Singh KhURANA
}

\begin{abstract}
For an Abelian, Archimedean Dedekind complete lattice-ordered, weakly $\sigma$-distributive group $G$ and an algebra $\mathcal{F}$ of subsets of a set $X$, every positive measure $\mu: \mathcal{F} \rightarrow G$ has a unique countably additive (in order convergence) extension to $\sigma$-algebra generated by $\mathcal{F}$.
\end{abstract}

\section{Introduction and notations}

In this note, $R$ is the set of real numbers and $\bar{R}=[-\infty, \infty], G$ is an Abelian, Archimedean Dedekind complete lattice-ordered, weakly $\sigma$-distributive group, $\mathcal{F}$ is an algebra of subsets of a set $X$ and $\mu: \mathcal{F} \rightarrow G$ a positive measure (this means for a disjoint sequence $\left\{A_{n}\right\} \subset \mathcal{F}$ with $\cup A_{n} \in \mathcal{F}, \mu(A)=\sum \mu\left(A_{n}\right)$, considering limit in order convergence) and $\mathcal{A}$ the $\sigma$-algebra generated by $\mathcal{F}$.

In [6], using Carathéodory measurability, the author has given a very interesting sophisticated proof of the unique extension, in order convergence, of the measure to $\mathcal{A}$ (in [4, 5] the author has given other proofs of this result). In this note we prove that this result can also be easily obtained from the representation theorem proved in [8] (see also [3]).

\section{Main result}

Theorem 1 ([6, Theorem 5.1]). Let $\mu: \mathcal{F} \rightarrow G$ be a positive measure. Then there is a unique extension $\mu: \mathcal{A} \rightarrow G$ which is positive and countably additive in order convergence.

2000 Mathematics Subject Classification: Primary 28B05; Secondary $46 \mathrm{G} 10$.

Keywords: extremely disconnected compact Hausdorff spaces, lattice-valued measures. 
Pr o of. For a compact Hausdorff Stonian (extremely disconnected) space $Y$, $\mathcal{E}(Y)$ will denote the space of all continuous functions $f: Y \rightarrow \bar{R}$ such that $f$ is finite-valued almost everywhere (this means the set where it is $\pm \infty$ is nowhere dense).

As in [8, we can assume, that $\mathcal{F}$ separates the points of $X$. As in [8, p. 70], there is a totally disconnected compact Hausdorff space $\tilde{X}$, the Boolean space for $\mathcal{F}$, in which $X$ is dense. If $\tilde{\mathcal{F}}$ and $\mathcal{B}$ are the classes of all clopen subsets and all Baire subsets of $\tilde{X}$, then the mapping $\rho: B \rightarrow B \cap X$, when restricted to $\tilde{\mathcal{F}}$, is an isomorphism onto $\mathcal{F}$ and when restricted to $\mathcal{B}$ is a $\sigma$-homomorphism of $\mathcal{B}$ onto a class which contains $\mathcal{A}$ with kernel being the class of Baire sets disjoint from $X$ [8]. The measure $\mu$ gives a finitely additive positive mapping

$$
\tilde{\mu}: \tilde{\mathcal{F}} \rightarrow G, \quad \tilde{\mu}(A)=\mu(A \cap X) .
$$

By [2, Theorem 4], there is a compact Hausdorff Stonian space $Y$ such that $G$ is lattice group isomorphic to a sub-lattice group of $\mathcal{E}(Y)$, preserving arbitrary sup and inf; we denote this isomorphism by $i$. Put $i \circ \mu(X)=e_{0}$ and let $Y_{0}$ be an open dense subset of $Y$ where $e_{0}$ is finite-valued. We denote by $C\left(Y_{0}\right)$ the space of all real-valued continuous functions on the locally compact space $Y_{0}$. The mapping $C\left(Y_{0}\right) \rightarrow \mathcal{E}(Y), f \rightarrow \tilde{f}$ (continuous extension) is a lattice isomorphism preserving arbitrary sup and inf. Put $e=\left(e_{0}\right)_{\mid Y_{0}}$. The finitely additive positive measure

$$
\nu: \tilde{\mathcal{F}} \rightarrow C\left(Y_{0}\right)
$$

is defined as

$$
\nu(A)=(i \circ \tilde{\mu}(A))_{\mid Y_{0}} .
$$

Putting

$$
I=\left\{f \in C\left(Y_{0}\right):|f| \leq e\right\}
$$

we get a Dedekind complete vector sub-lattice $E$ of $C\left(Y_{0}\right), E=\cup_{n=1}^{\infty} n I$ with a strong unit $e$ and a positive finitely additive measure $\nu: \tilde{\mathcal{F}} \rightarrow E$. Defining the norm $\|$.$\| on E,\|f\|=\inf \{\lambda>0:|f| \leq \lambda e\}, E$ becomes a Dedekind complete $M$-space [1] and so there is an extremely disconnected compact Hausdorff space $S$ such that $E$ is linear lattice isomorphic to $C(S)$, preserving arbitrary sup and inf. Thus we get a finitely additive positive measure $\nu: \tilde{\mathcal{F}} \rightarrow C(S)$ which extends to linear positive mapping

$$
\nu: \mathcal{S}(\tilde{\mathcal{F}}) \rightarrow C(S), \mathcal{S}(\tilde{\mathcal{F}})
$$

being the space of all real-valued $\tilde{\mathcal{F}}$-simple functions on $X$; with sup norm topology on $\mathcal{S}(\tilde{\mathcal{F}})$, this mapping is easily verified to be continuous. Since $X$ is totally disconnected, by Stone-Weierstrass theorem, $\mathcal{S}(\tilde{\mathcal{F}})$ is norm dense in $C(\tilde{X})$. Thus, we get a positive continuous mapping

$$
\nu: C(\tilde{X}) \rightarrow C(S) .
$$


By [7, we get a quasi-regular Baire measure on $\tilde{X}$ which extends $\nu$. Since $G$ is weakly $\sigma$-distributive, the measure $\nu: \mathcal{B} \rightarrow G$ is regular. Now, for any compact $G_{\delta}$-set $C \subset \tilde{X} \backslash X$, there is a decreasing sequence $\left\{F_{n}\right\} \subset \tilde{\mathcal{F}}$ such that $F_{n} \downarrow \chi_{C}$; since $\mu$ is a measure, we get $\nu(C)=0$. By regularity, $\nu(B)=0$ for any Baire subset of $\tilde{X}$ disjoint from $X$. Now, for any $A \in \mathcal{A}$ we can define $\mu(A)=\nu(B)$ for any Baire subset $B$ of $\tilde{X}$ with $B \cap X=A$. The rest is easily verified.

\section{REFERENCES}

[1] Aliprantis, C. D.-BurkinshaW, O.: Positive Operators. Academic Press, Orlando, 1985.

[2] BERNAU, S. J.: Unique representation of Archimedaen lattice groups and normal Archimedean lattice rings, Proc. London Math. Soc. (3) 15 (1965), 599-631.

[3] KHURANA, S. S.: Lattice-valued Borel measures, Rocky Mountain J. Math. 6 (1976), 377-382.

[4] RIEČAN, B.: On measures and integrals with values in ordered groups, Math. Slovaca 33 (1983), 153-163.

[5] RIEČAN, B.-NEUBRUnN, T.: Integral, Measure and Ordering. Math. Appl., Vol. 411, Kluwer Acad. Publ., Dordrecht, 1997.

[6] RIEČAN, B.: Carathéodory measurability revisited, Tatra Mt. Math. Publ. 34 (2006), 321-332.

[7] WRIGHT J. D. M.: Stone-algebra-valued measures and integrals, Proc. London Math. Soc. 19 (1969), 107-122.

[8] WRIGHT J. D. M.: The measure problem for vector lattices, Ann. Inst. Fourier (Grenoble) 21 (1971), 65-85. 